\title{
Vine performance of 'Italia' table grape in relation to organic soil amendment
}

\author{
L. Tarricone, G. Masi, S. Roccotelli, A.R. Caputo e P. Crupi \\ Consiglio per la ricerca in agricoltura e l'analisi dell'economia agraria di ricerca, Centro di ricerca Viticoltura ed Enologia, Via \\ Casamassima, 148, 70010 Turi (BA), Italia
}

\begin{abstract}
The following research took place during the 2018 - '19 season in 'Italia' table grape vineyard in Apulia. Four treatments were compared: no fertilized (T), organic amendment fertilized (B) at rate of 5.0 ton/hectare, equivalent at the rate of $100 \mathrm{Kg} \mathrm{Nha}^{-1}$, $75 \mathrm{Kg} \mathrm{P}_{2} \mathrm{O}_{5} \mathrm{ha}^{-1}$ and $75 \mathrm{Kg} \mathrm{K}_{20} 2 \mathrm{ha}^{-1}$; mineral fertilized (M) at the same rate of $\mathrm{N}, \mathrm{P}_{2} \mathrm{O}_{5}$ and $\mathrm{K}_{2} \mathrm{O}$ of organic fertilized vines and finally a cover crops treatment with a mixture of vetch, pea and lentil $(\mathrm{S})$. Leaf gas exchange during summer season shown significant differences in term of net $\mathrm{CO}_{2}$ assimilation, stomatal conductance, transpiration onto organic fertilized vines respect to control vines. Vines fertilized with organic amendment shown a better water status during warmest summer days since organic compounds improve the soil-water holding capacity. Compared to unfertilized vines, vines fertilized with organic amendment have shown a significant increase in yield per vine $(+32 \%)$ in relation to the highest cluster weight and the highest sugar content respect to mineral fertilized vines. Our results indicates that application of an high level of soil organic amendment on 'Italia' table grape vines increase grapevines yield, berry solid soluble solid and have improved soil properties, contrasting summer drought effect.
\end{abstract}

\section{Introduzione}

La conservazione e il miglioramento del contenuto di sostanza organica del suolo in vigneto rappresentano obiettivi chiave della resilienza dell' agroecosistema rispetto ai cambiamenti climatici e ai suoi effetti negativi sullo stato idrico e sulla fisiologia della vite $[1,3,5,6]$.

Vi è ancora una ridotta disponibilità di informazioni scientifiche sugli effetti dell'apporto di ammendanti organici sulle funzioni della vite e sulla resa per pianta in viticoltura da tavola $[2,4,7]$.

Al fine di approfondire aspetti legati all'impiego di ammendanti organici in viticoltura è stata impostata una ricerca in Puglia nel 2018-'19 in un vigneto commerciale ad uva da tavola della cv Italia, in agro di Casamassima (BA). Il vigneto è allevato a tendone con distanze di impianto di $2.40 \times 2.40 \mathrm{~m}$ (1736 viti/ettaro) ed è provvisto di impianto irriguo a micro-portata di erogazione.

Le tesi poste a confronto sono state le seguenti:

- suolo non concimato $(\mathrm{T})$;

- suolo con semina ( $80 \mathrm{Kg} / \mathrm{ha}$ ) di coltura leguminosa da sovescio (S) rappresentato da un miscuglio di veccia $(70 \%)$, lenticchia (15\%) e pisello $(15 \%)$;

- concimazione al suolo con apporto di ammendante compostato (B) alla dose di 5 ton/ettaro pari a 100 unità di azoto per ettaro, 75 unità di $\mathrm{P}_{2} \mathrm{O}_{5}$ e 75 unità di $\mathrm{K}_{2} \mathrm{O}$;

- concimazione al suolo con apporto di fertilizzanti minerali (M) semplici (azotato, fosfatico e potassico) con lo stesso apporto di unità $\mathrm{N}: \mathrm{P}_{2} \mathrm{O} 5$ e $\mathrm{K}_{2} \mathrm{O}$ della tesi $\mathrm{B}$.
L'ammendante compostato misto impiegato (BioVegetal, Tersan Puglia SPA, Modugno, Italia) ha un contenuto dichiarato di azoto organico del $2 \%, 1.5 \%$ di $\mathrm{P}_{2} \mathrm{O}_{5}$ e $1.5 \%$ di $\mathrm{K}_{2} \mathrm{O}$.

Eे stato adottato uno schema sperimentale a blocco randomizzato con tre ripetizioni per tesi (ogni ripetizione presenta una superficie di $207 \mathrm{~m}^{2}$ ).

L'apporto dell'ammendante e dei formulati minerali è stato effettuato nel periodo di riposo vegetativo del vigneto e la semina del miscuglio da sovescio nello stesso periodo.

\section{Materiali e metodi}

Ai fini della caratterizzazione del microclima della chioma in relazione al vigore eventualmente indotto dalle tecniche a confronto si è proceduto alla misura di diversi parametri quali l'indice Red:Far red, la radiazione ultravioletta UV A $(315-400 \mathrm{~nm})$ e UV B $(280-315 \mathrm{~nm})$ la radiazione fotosinteticamente attiva PAR $(400-700 \mathrm{~nm})$ e i valori termo-igrometrici della chioma.

La valutazione indiretta dello stato nutrizionale è stata effettuata attraverso la misura dell'indice in verde SPAD delle foglie con strumento portatile (SPAD 502, Minolta), dato la positiva correlazione tra indice SPAD, estratto di clorofilla e contenuto di azoto nelle foglie o correlazioni negative riguardo alla concentrazione fogliare di potassio.

Inoltre sono state eseguiti rilievi fogliari dell'indice di vegetazione NDVI (Normalized Difference Vegetation Index) calcolato dalla formula:

$$
\mathrm{NDVI}=\mathrm{NIR}-\mathrm{red} / \mathrm{NIR}+\mathrm{red}
$$


Tabella 1. Analisi fisico-chimiche del suolo.

\begin{tabular}{|c|c|c|c|c|c|c|c|c|}
\hline TESI & $\begin{array}{c}\text { Sabbia } \\
(\mathbf{g} / \mathbf{1 0 0} \\
\mathbf{g} \text { TF })\end{array}$ & $\begin{array}{c}\text { Limo } \\
(\mathbf{g} / \mathbf{1 0 0} \\
\mathbf{g} \text { TF) }\end{array}$ & $\begin{array}{c}\text { Argilla } \\
\mathbf{g} \text { TF }) \\
\mathbf{g} \text { TF })\end{array}$ & $\begin{array}{c}\text { Azoto } \\
\text { totale } \\
(\mathbf{g} / \mathbf{k g})\end{array}$ & Tessitura & $\begin{array}{c}\text { Anidride fosforica } \\
\text { assimilabile } \\
\text { (ppm) }\end{array}$ & $\begin{array}{c}\text { Potassio } \\
\text { scambiabile } \\
\text { (ppm) }\end{array}$ & $\begin{array}{c}\text { Sostanza organica } \\
\text { (g/100 } \\
\mathbf{g} \text { TF })\end{array}$ \\
\hline $\mathrm{T}$ & 31.6 & 30.7 & 37.7 & 1.88 & Limo-argillosa & 79 & 542 & 3.17 \\
\hline $\mathrm{B}$ & 30 & 28.3 & 41.7 & 2.03 & Argillosa & 109 & 654 & 3.43 \\
\hline $\mathrm{M}$ & 29.6 & 28.7 & 41.7 & 2.05 & Argillosa & 63 & 518 & 3.46 \\
\hline $\mathrm{S}$ & 30 & 30.3 & 39.7 & 1.86 & Limo-argillosa & 72 & 1359 & 3.11 \\
\hline
\end{tabular}

Tabella 2. Indici di fertilità del germoglio nella cv Italia.

\begin{tabular}{|c|c|c|c|c|c|c|}
\hline TESI & $\begin{array}{c}\text { N. gemme } \\
(\mathbf{n})\end{array}$ & $\begin{array}{c}\text { N. germogli } \\
(\mathbf{n})\end{array}$ & $\begin{array}{c}\text { N. grappoli } \\
(\mathbf{n})\end{array}$ & $\begin{array}{c}\text { N. grappoli// } \\
\text { N. gemme }\end{array}$ & $\begin{array}{c}\text { N./n grappoli. } \\
\text { germogli }\end{array}$ & $\begin{array}{c}\% \\
\text { germogliamento }\end{array}$ \\
\hline $\mathrm{T}$ & 32.22 & 24 & 35.33 & 1.1 & 1.46 & 74.8 \\
\hline $\mathrm{B}$ & 38.89 & 27 & 43.89 & 1.12 & 1.61 & 69.5 \\
\hline $\mathrm{M}$ & 33.22 & 25 & 35.11 & 1.05 & 1.4 & 75.56 \\
\hline $\mathrm{S}$ & 35.22 & 25.33 & 39.11 & 1.1 & 1.54 & 71.47 \\
\hline
\end{tabular}

Tabella 3. Caratterizzazione del microclima della chioma.

\begin{tabular}{|c|c|c|c|c|c|c|c|c|}
\hline \multirow{3}{*}{ TESI } & \multirow{3}{*}{ SPAD } & \multirow{3}{*}{ NDVI } & \multirow{3}{*}{ R:FR } & UV A & UV B & PAR & \multirow{3}{*}{$\begin{array}{c}\text { Umidità } \\
\text { relativa (\%) }\end{array}$} & Temperatura \\
\hline & & & & $\left(\mathrm{mW} \mathrm{m}^{-2}\right)$ & $\left(\mathrm{mW} \mathrm{m} \mathbf{m}^{-2}\right)$ & 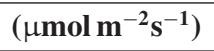 & & chioma \\
\hline & & & & & & & & $\left({ }^{\circ} \mathbf{C}\right)$ \\
\hline $\mathrm{T}$ & $37.96 \mathrm{c}$ & 0.77 & $0.40 \mathrm{~b}$ & $723.73 \mathrm{c}$ & $43.50 \mathrm{c}$ & $544.9 \mathrm{c}$ & $40.77 \mathrm{a}$ & $35.17 \mathrm{~b}$ \\
\hline B & $40.61 \mathrm{ab}$ & 0.76 & $0.29 \mathrm{c}$ & $462.94 \mathrm{c}$ & $34.22 \mathrm{c}$ & $431.9 \mathrm{c}$ & $38.18 \mathrm{~b}$ & $35.43 \mathrm{a}$ \\
\hline$M$ & $42.37 \mathrm{a}$ & 0.8 & $0.25 \mathrm{c}$ & $419.89 \mathrm{c}$ & $15.21 \mathrm{c}$ & $467.9 \mathrm{c}$ & $41.56 \mathrm{a}$ & $35.0 \mathrm{~b}$ \\
\hline$S$ & $39.60 \mathrm{bc}$ & 0.82 & $0.36 \mathrm{bc}$ & $787.27 \mathrm{c}$ & $37.77 \mathrm{c}$ & $518.1 \mathrm{c}$ & $41.05 \mathrm{a}$ & $35.04 \mathrm{~b}$ \\
\hline Esterno & & & $1.20 \mathrm{a}$ & $21708.8 \mathrm{a}$ & $1713.75 \mathrm{a}$ & $1856.70 \mathrm{a}$ & $31.29 \mathrm{c}$ & \\
\hline Sotto film plastico & & & & $7708.34 \mathrm{~b}$ & $277.68 \mathrm{~b}$ & $1013.90 \mathrm{~b}$ & & \\
\hline
\end{tabular}

Lettere diverse indicano significatività a $P<0.05$ per SNK test.

dove NIR (da 700 a $1100 \mathrm{~nm}$ ) e red (da 400 a $700 \mathrm{~nm}$ ) corrispondono rispettivamente al valore di riflettanza nelle due diverse lunghezze d'onda del visibile (rosso) e nel vicino infrarosso, e per il quale all' aumentare della vigore vegetativo si registrano incrementi di NDVI nel tipico range $0+1$. L'indice NDVI è noto essere correlato con la quantità di biomassa per unità di superficie fogliare (LAI) e con il vigore del vigneto (peso del legno di potatura).

Ai fini della valutazione dello stato fisiologico delle viti nelle diverse tesi sono state effettuate misurazioni dell'intensità degli scambi gassosi per unità di superficie fogliare (assimilazione carbonica netta, conduttanza stomatica, traspirazione, efficienza d'uso dell'acqua) mediante misuratore portatile a raggi infrarossi (IRGA IFL, ADC Bioscientific Ltd., Hoddeston, UK) con rilievi effettuati nelle ore centrali del giorno, in alcune fasi fenologiche della vite. I rilievi sono stati eseguiti su foglie mediane di germogli esposti alla luce con la camera fogliare orientata a un flusso fotonico di $1500 \mu \mathrm{mol} \mathrm{m} \mathrm{m}^{-2} \mathrm{~s}^{-1} \mathrm{di}$ PAR (radiazione fotosinteticamente attiva). Inoltre sono state eseguite nelle quattro tesi misure di scambi gassosi del suolo con Soil Respiration Chamber (EGM-5 Portable $\mathrm{CO}_{2}$ Gas Analyzer, PP Systems, USA).

Alla raccolta commerciale della varietà Italia sono state valutate su campioni rappresentativi di grappoli le caratteristiche carpometriche e la composizione chimica del succo (grado rifrattometrico, acidità titolabile e $\mathrm{pH}$ ).

Inoltre, campioni di 20 acini per tesi, sono stati sottoposti a determinazione delle caratteristiche reologiche, con Testing Machine Texture Analyzer (Zwick/Roell mod. BT1) determinando i seguenti parametri:

- resistenza alla compressione o durezza $(\mathrm{N})$, valore massimo della forza dell'acino durante il primo ciclo di compressione;

- coesività $(\mathrm{N})$, forza dei legami interni che "riformano" la struttura dell'acino;

- elasticità (mm), altezza che l'acino recupera durante il tempo che trascorre tra la fine del primo ciclo e l'inizio del secondo;

- gommosità $(\mathrm{N})$, energia richiesta per disintegrare un cibo semisolido (acino) fino a quando è pronto per la deglutizione;

- masticabilità $(\mathrm{J})$, energia richiesta per masticare un cibo solido (acino) fino a quando è pronto per la deglutizione.

Il colore della bacca è stato misurato con un colorimetro a riflettanza da laboratorio (sistema CIEL*a*b*) eseguendo le letture sui due lati di 50 acini per tesi, delle coordinate 
Tabella 4. Fluorescenza della clorofilla (luglio 2018).

\begin{tabular}{|c|c|c|c|c|c|c|c|c|c|}
\hline TESI & PAR & $\begin{array}{l}\text { Temperatura } \\
\text { fogliare }\left({ }^{\circ} \mathbf{C}\right)\end{array}$ & $\begin{array}{c}\text { Temperatura } \\
\text { intorno chioma }\left({ }^{\circ} \mathrm{C}\right)\end{array}$ & $\begin{array}{c}\text { Umidità relativa } \\
\text { ambiente }(\%)\end{array}$ & Fs & Fms & $\mathbf{Y}$ & \begin{tabular}{|c|} 
ETR \\
$\left(\mu \mathrm{mol}\right.$ elettroni $\left.\mathbf{m}^{-2} \mathbf{s}^{-1}\right)$
\end{tabular} & alpha \\
\hline $\mathrm{T}$ & 697.92 & 30.97 & $32.85 \mathrm{c}$ & 44.33 & 311.75 & 672.08 & 0.515 & 145.24 & 0.88 \\
\hline B & 586.33 & 31.17 & $33.17 \mathrm{a}$ & 44.92 & 306.5 & 699.75 & 0.536 & 119.04 & 0.87 \\
\hline $\mathrm{M}$ & 901.83 & 31.72 & $32.47 \mathrm{~d}$ & 44.17 & 275.58 & 522 & 0.454 & 170.67 & 0.86 \\
\hline$S$ & 662.67 & 31.3 & $33.04 \mathrm{~b}$ & 44.5 & 299.5 & 632.25 & 0.494 & 139.44 & 0.87 \\
\hline
\end{tabular}

Lettere diverse indicano significatività a $P<0.05$ per SNK test.

Tabella 5. Scambi gassosi fogliari (luglio 2018).

\begin{tabular}{|c|c|c|c|c|c|c|}
\hline TESI & $\begin{array}{l}\text { Temperatura } \\
\text { fogliare }\left({ }^{\circ} \mathbf{C}\right) \\
\end{array}$ & $\begin{array}{c}\text { Traspirazione } \\
\left(\mathrm{mol} \mathrm{m}^{-2} \mathbf{s}^{-1}\right)\end{array}$ & \begin{tabular}{|c|}
$\begin{array}{c}\text { Conduttanza stomatica } \\
\left(\mathrm{mol} \mathrm{m}^{-2} \mathbf{s}^{-1}\right)\end{array}$ \\
\end{tabular} & \begin{tabular}{|c|} 
Fotosintesi netta \\
$\left(\mu \mathrm{mol} \mathrm{m} \mathbf{m}^{-2} \mathrm{~s}^{-1}\right)$
\end{tabular} & $\begin{array}{c}\text { A/E - WUEintrinseca } \\
\left(\mu \mathrm{mol}^{-1} \mathrm{CO}_{2} \mathrm{mmol} \mathrm{H}_{2} \mathrm{O}\right)\end{array}$ & $\begin{array}{c}\text { A/gs - WUEistantanea } \\
\left(\mu \mathrm{mol} \mathrm{CO} \mathrm{CO}_{2} / \mathrm{mol} \mathrm{H}_{2} \mathrm{O}\right)\end{array}$ \\
\hline$T$ & $34.23 \mathrm{c}$ & $1.67 \mathrm{~b}$ & $0.07 \mathrm{~b}$ & $6.53 \mathrm{ab}$ & 3.9 & 97.82 \\
\hline$B$ & $33.97 \mathrm{~d}$ & $2.42 \mathrm{a}$ & $0.11 \mathrm{a}$ & $7.93 \mathrm{a}$ & 3.26 & 74.04 \\
\hline$M$ & $34.57 \mathrm{~b}$ & $1.42 \mathrm{bc}$ & $0.05 \mathrm{bc}$ & $5.05 \mathrm{ab}$ & 3.55 & 93.69 \\
\hline$S$ & $34.87 \mathrm{a}$ & $1.16 \mathrm{c}$ & $0.04 \mathrm{c}$ & $3.53 \mathrm{~b}$ & 3.06 & 88.17 \\
\hline
\end{tabular}

Lettere diverse indicano significatività a $P<0.05$ per SNK test.

Tabella 6. Caratterizzazione del microclima della chioma e indici fogliari (luglio 2018).

\begin{tabular}{|c|c|c|c|c|c|}
\hline \multirow{2}{*}{ TESI } & \multirow{2}{*}{ SPAD } & \multirow{2}{*}{ NDVI } & \multirow{2}{*}{$\mathbf{R}: \mathbf{F R}$} & Umidità relativa & Temperatura \\
\cline { 5 - 6 } & & & & chioma & chioma \\
\cline { 5 - 6 } & & & $\mathbf{( \% )}$ & $\left({ }^{\mathbf{C}} \mathbf{)}\right.$ \\
\hline $\mathrm{T}$ & $40.24 \mathrm{c}$ & 0.68 & 0.33 & $40.77 \mathrm{a}$ & $35.17 \mathrm{bc}$ \\
\hline $\mathrm{B}$ & $42.47 \mathrm{~b}$ & 0.67 & 0.24 & $38.18 \mathrm{~b}$ & $35.43 \mathrm{~b}$ \\
\hline $\mathrm{M}$ & $44.14 \mathrm{a}$ & 0.73 & 0.2 & $41.56 \mathrm{a}$ & $35.0 \mathrm{c}$ \\
\hline $\mathrm{S}$ & $41.76 \mathrm{~b}$ & 0.74 & 0.36 & $41.05 \mathrm{a}$ & $35.04 \mathrm{c}$ \\
\hline Esterno & & & & $31.29 \mathrm{c}$ & $36.9 \mathrm{a}$ \\
\hline
\end{tabular}

Lettere diverse indicano significatività a $P<0.05$ per SNK test.

cromatiche L* (luminosità o luminanza, espressa in percentuale ossia 0 per il nero e 100 per il bianco; $\mathrm{a}^{*}$ (misura del range di colore dal verde [-] al rosso $[+]$ e $b^{*}$ (misura del range di colore dal blu [-] al giallo $[+]$.

Successivamente alla raccolta è stata determinata la superficie fogliare totale per vite mediante misura in laboratorio con $\mathrm{Li} 3100 \mathrm{C}$ Leaf area meter (LI-COR Environmental, USA).

I dati raccolti sono stati sottoposti ad analisi della varianza e separazione delle medie con test SNK.

\section{Risultati e discussione}

Le analisi fisico-chimiche del suolo hanno evidenziato una tessitura limo-argillosa, con $\mathrm{pH}$ alcalino, una sufficiente dotazione di azoto, fosforo assimilabile e potassio scambiabile (Tabella 1).

Il carico di gemme delle viti mediamente ripartito su 4 capi a frutto per vite ha evidenziato una percentuale di germogliamento compresa tra il 70-75\% e indici di fertilità del germoglio statisticamente non diversi e compresi tra 1.40-1.60, tipici della varietà Italia (Tabella 2).

Il maggiore vigore indotto dalla concimazione minerale si è tradotto in un valore maggiore dell' indice fogliare SPAD mentre la tesi test non concimata ha espresso il valore minore $(-10 \%)$.
L'indice NDVI non ha evidenziato differenze significative mentre l'indice Red:Far red, indicativo del grado di ombreggiamento della chioma ha evidenziato i valori minori indicativi di chiome più dense nelle tesi $\mathrm{M}$ e $\mathrm{B}$. Non sono emerse differenze in termini intercettazione a livello di chioma sia della radiazione ultravioletta UV A e UV B che della radiazione fotosinteticamente attiva. I valori minori di umidità relativa della chioma sono stati riscontrati nelle viti della tesi B caratterizzata da un maggiore valore della temperatura (Tabella 3 ).

Gli scambi gassosi fogliari effettuati in post allegagione non hanno evidenziato differenze significative in termini di fluorescenza della clorofilla, tranne un valore maggiore della temperatura a livello di chioma nella tesi B. Invece i parametri di funzionalità fogliare hanno evidenziato differenze significative per fotosintesi, traspirazione, conduttanza stomatica. Nello specifico le viti della tesi B hanno espresso maggiori tassi di traspirazione, conduttanza stomatica e fotosintesi netta. (Tabella 4 e 5).

La caratterizzazione del microclima della chioma a luglio ha evidenziato un maggiore valore dell'indice fogliare SPAD nella tesi con apporto di fertilizzante minerale (M), valori simili dell'indice NDVI e del rapporto R:FR. A livello di microclima della chioma, minore umidità relativa è stata riscontrata nella chioma di viti della tesi B mentre i valori termici minori si sono riscontrati nelle viti delle tesi $\mathrm{M}$ e S (Tabella 6). 
Tabella 7. Scambi gassosi fogliari in invaiatura.

\begin{tabular}{|c|c|c|c|c|c|c|}
\hline TESI & $\begin{array}{l}\text { Temperatura } \\
\text { fogliare }\left({ }^{\circ} \mathbf{C}\right)\end{array}$ & $\begin{array}{c}\text { Traspirazione } \\
\left(\mathrm{mol} \mathrm{m}^{-2} \mathbf{s}^{-1}\right)\end{array}$ & $\begin{array}{c}\text { Conduttanza stomatic } \\
\left(\mathrm{mol} \mathrm{m}^{-2} \mathrm{~s}^{-1}\right)\end{array}$ & $\begin{array}{c}\text { Fotosintesi netta } \\
\left(\mu \mathrm{mol} \mathrm{m} \mathbf{m}^{-2} \mathbf{s}^{-1}\right)\end{array}$ & $\begin{array}{c}\text { A/E - WUEintrinseca } \\
\left(\mu \mathrm{mol} \mathrm{CO}_{2} \mathrm{mmol}^{-1} \mathrm{H}_{2} \mathrm{O}\right)\end{array}$ & $\begin{array}{l}\text { A/gs - WUEistantanea } \\
\left(\mu \mathrm{mol} \mathrm{CO}{ }_{2} \mathrm{mmol}^{-1} \mathrm{H}_{2} \mathrm{O}\right)\end{array}$ \\
\hline $\mathrm{T}$ & $33.57 \mathrm{a}$ & 1.72 & 0.07 & 4.58 & 3.09 & 83.52 \\
\hline B & $34.30 \mathrm{a}$ & 2.17 & 0.097 & 5.7 & 2.61 & 63.56 \\
\hline $\mathrm{M}$ & $34.33 \mathrm{a}$ & 2 & 0.08 & 5.2 & 2.63 & 66.03 \\
\hline S & $31.70 \mathrm{~b}$ & 2.46 & 0.143 & 9.61 & 3.91 & 68.37 \\
\hline
\end{tabular}

Lettere diverse indicano significatività a $P<0.05$ per SNK test.

Tabella 8. Scambi gassosi del suolo all'invaiatura.

\begin{tabular}{|c|c|c|c|}
\hline TESI & Temperatura suolo $\left({ }^{\circ} \mathbf{C}\right)$ & Temperatura aria $\left({ }^{\circ} \mathbf{C}\right)$ & Respirazione del suolo $\left(\mathbf{g ~ C O}_{2} \mathbf{m}^{-2} \mathbf{h}^{-1}\right)$ \\
\hline $\mathrm{T}$ & $25.47 \mathrm{a}$ & $34.82 \mathrm{~b}$ & $0.12 \mathrm{~b}$ \\
\hline $\mathrm{B}$ & $24.47 \mathrm{~b}$ & $33.98 \mathrm{c}$ & $0.30 \mathrm{a}$ \\
\hline $\mathrm{M}$ & $25.16 \mathrm{ab}$ & $35.30 \mathrm{a}$ & $0.15 \mathrm{~b}$ \\
\hline $\mathrm{S}$ & $24.40 \mathrm{~b}$ & $34.97 \mathrm{~b}$ & $0.30 \mathrm{a}$ \\
\hline
\end{tabular}

Lettere diverse indicano significatività a $P<0.05$ per SNK test.

Tabella 9. Scambi gassosi fogliari a maturazione.

\begin{tabular}{|c|c|c|c|c|c|c|}
\hline TESI & $\begin{array}{l}\text { Temperatura } \\
\text { fogliare }\left({ }^{\circ} \mathbf{C}\right)\end{array}$ & $\begin{array}{c}\text { Traspirazione } \\
\left(\mathrm{mol} \mathrm{m}^{-2} \mathrm{~s}^{-1}\right)\end{array}$ & $\begin{array}{c}\text { Conduttanza stomatica } \\
\left(\mathrm{mol} \mathrm{m}^{-2} \mathbf{s}^{-1}\right)\end{array}$ & 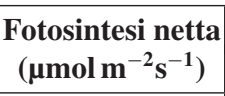 & $\begin{array}{c}\text { A/E - WUEintrinseca } \\
\left(\mu \mathrm{mol} \mathrm{CO} \mathrm{mmol}^{-1} \mathrm{H}_{2} \mathrm{O}\right)\end{array}$ & $\begin{array}{c}\text { A/gs - WUEistantanea } \\
\left(\mu \mathrm{mol} \mathrm{CO} \text { mmol }^{-1} \mathbf{H}_{2} \mathrm{O}\right)\end{array}$ \\
\hline $\mathrm{T}$ & $33.63 \mathrm{a}$ & $3.23 \mathrm{a}$ & $0.16 \mathrm{a}$ & $6.68 \mathrm{ab}$ & $2.07 \mathrm{ab}$ & $41.77 \mathrm{~b}$ \\
\hline B & $31.93 \mathrm{c}$ & $2.66 \mathrm{a}$ & $0.15 \mathrm{a}$ & $7.75 \mathrm{a}$ & $2.90 \mathrm{a}$ & $54.75 \mathrm{ab}$ \\
\hline M & $34 \mathrm{a}$ & $2.32 \mathrm{a}$ & $0.10 \mathrm{a}$ & $3.93 \mathrm{bc}$ & $1.62 \mathrm{~b}$ & $39.08 \mathrm{~b}$ \\
\hline S & $32.90 \mathrm{~b}$ & $0.99 \mathrm{~b}$ & $0.03 \mathrm{~b}$ & $2.46 \mathrm{c}$ & $2.56 \mathrm{a}$ & $73.06 \mathrm{a}$ \\
\hline
\end{tabular}

Lettere diverse indicano significatività a $P<0.05$ per SNK test.

Gli scambi gassosi fogliari effettuati all'invaiatura non hanno evidenziato differenze significative sui parametri di funzionalità fogliare quale fotosintesi, traspirazione, conduttanza stomatica e water use efficiency (Tabella 7).

Gli scambi gassosi del suolo in invaiatura hanno evidenziato invece un maggiore attività respiratoria legata sia ai microrganismi che all' attività radicale nelle tesi $\mathrm{B}$ e $\mathrm{S}$ in relazione all'apporto di sostanza organica legata alla concimazione nella tesi $\mathrm{B}$ e al sovescio delle leguminose nella tesi S (Tabella 8).

Non sono emerse differenze significative per l'indice fogliare SPAD all'invaiatura mentre l'indice NDVI è risultato minore nella tesi $\mathrm{B}$.

Gli scambi gassosi fogliari effettuati alla fine di settembre hanno evidenziato differenze significative sui parametri di funzionalità fogliare quale fotosintesi, traspirazione, conduttanza stomatica e water use efficiency. Nello specifico le viti delle tesi T, B e M hanno espresso maggiori tassi di traspirazione e conduttanza stomatica. Tuttavia le viti della tesi B hanno evidenziato maggiore assimilazione fotosintetica e WUE $\mathrm{W}_{\text {intrinseca }}$ (Tabella 9).

Gli scambi gassosi del suolo effettuati a ottobre alla maturazione hanno espresso minore attività respiratoria legata sia ai microrganismi che all'attività radicale nelle tesi B e M.

Alla raccolta il minore peso del grappolo (-32\%) è stato riscontrato nella tesi $\mathrm{T}$ non concimata. I valori maggiori si sono avuti nelle tesi $\mathrm{B}$ e $\mathrm{M}$ mentre il peso grappolo nella tesi $\mathrm{S}$ ha raggiunto un valore intermedio
$(-18 \%)$. Di conseguenza il valore maggiore del peso bacca si è riscontrato nella tesi $\mathrm{B}$ mentre le restanti tesi hanno presentato i i seguenti decrementi: tesi $\mathrm{T}(-5 \%)$, tesi $\mathrm{M}$ $(-13 \%)$, tesi S $(-12 \%)$. Le bacche dei grappoli della tesi B si sono caratterizzate per un valore maggiore del diametro polare (Tabella 10).

Da un punto di vista della composizione chimica minore grado rifrattometrico delle uve è stato riscontrato nella tesi $\mathrm{M}(-7 \%)$ rispetto alla tesi Test, in relazione alla pronta disponibilità dell'azoto minerale cha ha spinto sull'attività vegetativa. Il maggiore grado di maturazione delle uve è stato riscontrato nella tesi $\mathrm{T}$ e nella tesi B con un grado Brix prossimo ai 19. Se si considera l'indice di maturazione espresso dal rapporto grado rifrattometrico/acidità titolabile la tesi non concimata $\mathrm{T}$ ha raggiunto il maggiore grado di maturità delle uve seguita dalla tesi B (Tabella 10).

Da considerare che dal punto di vista della resa per vite la minore produzione per pianta si è registrata nella tesi testimone T non fertilizzata.

Riguardo alle caratteristiche fisiche dell'acino maggiore croccantezza (firmness) e resistenza al distacco dell'acino dal pedicello si sono riscontrate nella tesi B. Gli acini della tesi $\mathrm{M}$ hanno evidenziato rispettivamente una riduzione della firmness $(-27 \%)$ e della resistenza al distacco $(-31 \%)$ rispetto alla tesi B (Tabella 11).

Gli acini delle viti della tesi B si sono caratterizzati per un maggiore indice di giallo $\left(\mathrm{a}^{*}\right)$ e un valore maggiore del parametro croma $\left(a^{2}+b^{2}\right)^{0.5}$ mentre gli acini meno colorati sono apparsi essere quelli della tesi S (Tabella 11). 
Tabella 10. Caratteristiche carpometriche della cv Italia.

\begin{tabular}{|c|c|c|c|c|c|c|c|c|}
\hline \multirow[t]{2}{*}{ Tesi } & $\begin{array}{c}\text { Peso } \\
\text { grappolo }\end{array}$ & $\begin{array}{c}\text { Peso } \\
\text { bacca }\end{array}$ & $\begin{array}{l}\text { Diametro } \\
\text { polar }\end{array}$ & $\begin{array}{c}\text { Diametro } \\
\text { equatoriale }\end{array}$ & $\begin{array}{c}\text { Grado } \\
\text { alerifrattometric }\end{array}$ & \multirow[t]{2}{*}{$\mathrm{pH}$} & Aciditá & \multirow[t]{2}{*}{$\begin{array}{c}\text { Grado } \\
\text { rifrattometrico/ac.tito }\end{array}$} \\
\hline & (g) & (g) & $(\mathbf{m m})$ & $(\mathbf{m m})$ & $\left({ }^{\circ}\right.$ Brix $)$ & & $(\mathrm{g} / \mathrm{L})$ & \\
\hline $\mathrm{T}$ & $501.4^{\mathrm{b}}$ & $9.33^{\mathrm{ab}}$ & $14.83^{\mathrm{a}}$ & 11.42 & $18.7^{\mathrm{a}}$ & $3.83^{\mathrm{a}}$ & $4.13^{\mathrm{c}}$ & $45.42 \mathrm{a}$ \\
\hline B & $753.7^{\mathrm{a}}$ & $9.84^{\mathrm{a}}$ & $14.93^{\mathrm{a}}$ & 11.61 & $18.7^{\mathrm{a}}$ & $3.69^{\mathrm{bc}}$ & $4.58^{\mathrm{b}}$ & $40.88 \mathrm{~b}$ \\
\hline $\mathrm{M}$ & $724.4^{\mathrm{a}}$ & $8.47^{\mathrm{c}}$ & $14.02^{b}$ & 11.22 & $17.3^{\mathrm{c}}$ & $3.72^{\mathrm{b}}$ & $4.90^{\mathrm{a}}$ & $35.91 \mathrm{c}$ \\
\hline S & $605.5^{\mathrm{ab}}$ & $8.62^{\mathrm{bc}}$ & $14.21^{\mathrm{ab}}$ & 11.21 & $18.1^{\mathrm{b}}$ & $3.66^{\mathrm{d}}$ & $5.05^{\mathrm{a}}$ & $35.33 \mathrm{c}$ \\
\hline P-level & $* *$ & $* * *$ & $* *$ & ns & $* * *$ & $* * *$ & $* * *$ & $* * *$ \\
\hline
\end{tabular}

Medie separate mediante test di Tukey. ${ }^{*} p<0.05 ;{ }^{* *} p<0.01 ;{ }^{* * *} p<0.001$.

Tabella 11. Caratteristiche reologiche dell'acino.

\begin{tabular}{|c|c|c|c|c|}
\hline TESI & $\mathbf{L}^{*}$ & $\mathbf{a}^{*}$ & $\mathbf{b}^{*}$ & $\mathbf{C}$ \\
\hline $\mathrm{T}$ & $35.62^{\mathrm{a}}$ & $-1.78^{\mathrm{a}}$ & $4.84^{\mathrm{ab}}$ & $5.21^{\mathrm{ab}}$ \\
\hline $\mathrm{B}$ & $33.52^{\mathrm{b}}$ & $-1.94^{\mathrm{b}}$ & $5.17^{\mathrm{a}}$ & $5.55^{\mathrm{a}}$ \\
\hline $\mathrm{M}$ & $34.31^{\mathrm{b}}$ & $-1.77^{\mathrm{a}}$ & $4.52^{\mathrm{b}}$ & $4.90^{\mathrm{b}}$ \\
\hline $\mathrm{S}$ & $34.52^{\mathrm{b}}$ & $-1.85^{\mathrm{ab}}$ & $3.90^{\mathrm{c}}$ & $4.37^{\mathrm{c}}$ \\
\hline P-level & $* * *$ & $* *$ & $* * *$ & $* * *$ \\
\hline
\end{tabular}

Medie separate mediante test di Tukey. ${ }^{*} p<0.05 ;{ }^{* *} p<0.01 ;{ }^{* * *} p<0.001$.

Tabella 12. Indici di equilibrio vegeto-produttivo nella cv Italia.

\begin{tabular}{|c|c|c|c|}
\hline TESI & $\begin{array}{l}\text { Produzione } \\
\text { per vite } \\
\left(\mathrm{Kg} \mathrm{vite}^{-1}\right)\end{array}$ & $\begin{array}{c}\text { Area } \\
\text { fogliare } \\
\left(\mathbf{m}^{2} \text { vite }^{-1}\right)\end{array}$ & $\begin{array}{l}\text { Area fogliare/ } \\
\text { produzione per } \\
\text { vite }\left(\mathrm{m}^{2} \mathrm{~kg}^{-1}\right)\end{array}$ \\
\hline $\mathrm{T}$ & $17.71 \mathrm{~d}$ & 19.92 & 1.13 \\
\hline B & $26.08 \mathrm{a}$ & 18.05 & 0.69 \\
\hline M & $25.43 \mathrm{~b}$ & 19.79 & 0.79 \\
\hline$S$ & $20.05 \mathrm{c}$ & 10.98 & 0.55 \\
\hline
\end{tabular}

Lettere diverse indicano significatività a $P<0.05$ per SNK test.

I rilievi di scambio gassoso del suolo effettuati nel periodo di riposo vegetativo (dicembre) hanno accertato un maggiore intensità di scambi gassosi di $\mathrm{CO}_{2}$ nelle tesi $\mathrm{T}$ e minerale $\mathrm{M}$.

Riguardo agli indici di equilibrio vegeto-produttivo la minore resa per vite si è ottenuta nelle viti della tesi $\mathrm{T}$ non concimata mentre nelle altre tesi si è assistito a un incremento dell' $47 \%$ della produzione per vite nella tesi $\mathrm{B}$, del $43 \%$ nella tesi $\mathrm{M}$ e del $13 \%$ nella tesi $\mathrm{S}$ rispetto a viti non fertilizzate.

L'area fogliare ha raggiunto valori statisticamente non diversi tra le tesi (pur evidenziandosi un trend alla riduzione nella tesi $\mathrm{S}$ ) e sviluppando un area per $\mathrm{kg}$ di uva prodotta compresa tra $0.6-1.1 \mathrm{~m}^{2} \mathrm{~kg}$ uva prodotta (Tabella 12).

Lo studio delle correlazioni tra le variabili considerate ha evidenziato positive correlazioni per parametri diversi quali la croma con l'indice di giallo $\left(\mathrm{b}^{*}\right)$ masticabilità verso gommosità, coesione verso elasticità, firmness verso la gommosità.

\section{Considerazioni conclusive}

I primi risultati della ricerca hanno evidenziato un effetto positivo dell'apporto dell'ammendante alle dosi testate sulle caratteristiche carpometriche (produzione per pianta, peso grappolo, peso acino) sul livello di maturazione delle uve, sui parametri fisici dell'acino quali la croccantezza ed infine sulla colorazione della buccia (incremento della componente gialla).

Inoltre l'apporto di sostanza organica al suolo attraverso l'ammendante o la pratica del sovescio ha determinato dei tassi di scambi gassosi di $\mathrm{CO}_{2}$ del suolo maggiori sino alla fase di invaiatura nelle tesi $\mathrm{B}$ e $\mathrm{S}$.

\section{Riferimenti}

[1] N. Bonilla, J.A. Gutiérrez-Barranquero, A. de Vicente, F.M. Cazorla, Diversity 4, 17 (2012)

[2] J.C. Fourie, S. Afr. J. Enol. Vitic. 32, 11 (2010)

[3] A.A.B Masoud, Res. J. Agric. Biol. Sci. 8, 9 (2012)

[4] F. Obriot, M. Stauffer, Y. Goubard, A. Revallier, L. Vieublé-Gonod, S. Houot, Acta Hort. 1146, 9 (2016)

[5] G. Ponchia, A. Bozzolo, F. Tateo, G. Concheri, Acta Hort. 938, 8 (2012)

[6] L. Rahman, A.M.A. Whitelaw-Weckert, B. Orchard, Soil Res. 52, 16 (2014)

[7] L. Tarricone, D. Di Gennaro, G. Debiase, A.M. Amendolagine, G. Gentilesco, G. Masi, Acta Hortic. 1217, 8 (2018) 